\title{
Pheromone Based Dynamic Vaccination for Immune Algorithms
}

\author{
Yutao Qi, Fang Liu, and Licheng Jiao \\ Institute of Intelligent Information Processing, Xidian University, Xi' an, China, 710071 \\ \{qi_yutao,f63liu\}@163.com \\ lchjiao@mail.xidian.edu.cn
}

\begin{abstract}
The vaccination which is a most effective protection against the virus is also a helpful part of the artificial immune systems (AIS). A vaccination in AIS means modifying some genes of an antibody in accordance with priori knowledge so as to gain higher affinity with greater probability. Since vaccination is problem specific, what can we do if we have no idea about the problem? To address this, we propose a pheromone based dynamic vaccination for real coded immune algorithms in this paper. The pheromone which is a term of the ant colony system is used as a carrier of knowledge learned from population's evolution and acts as a producer of dynamic vaccines. Experiments on numerical optimization problems indicate that the pheromone based vaccination operator has the ability of acquiring useful information about the objective functions and developing effective vaccines dynamically.
\end{abstract}

\section{Introduction}

Artificial immune systems (AIS) which can be defined as computational systems inspired by theoretical immunology and observed immune functions has attracted significant research interest over the years [1]. Inspired by the clonal selection theory, De Castro pioneered the Clonal selection algorithm (CSA) [2] in 2000. After that, many clonal selection based artificial immune algorithms have been proposed. The dynamic clonal selection algorithm (DynamiCS) constructed by Kim in 2002 [3] and polyclonal strategy proposed by Licheng Jiao in 2003 [4] are two of the most outstanding contributions. Lei Wang and Licheng Jiao introduced immune concepts and methods into evolutionary algorithm to form immune evolutionary algorithms [5]. In this work, two immune operators of vaccination and immune selection are designed and they have been proven to be able to restrain the degenerate phenomenon of GA.

Vaccination is a means of stimulating the immune system to produce diseasepreventing antibodies. Vaccines can be regarded as a priori knowledge about a certain disease. In the study of artificial immune systems, vaccines have been designed for different problems such as numerical optimization problem [6], TSP problem [7], SAR image segmentation [8] and intrusion detection problem [9] and so on. These vaccines are problem specific. What can we do when we have no idea about the problem? This paper is dealing with an approach for preparing vaccines adaptively when we know nothing about the character of the problems dealt with. 
This work proposed a pheromone based dynamic vaccination for immune algorithms to learn valuable information from population's evolution by stimulating the pheromone deposition and volatilization mechanism of ant colonies. In addition, the acquired knowledge is used to produce dynamic vaccines which act as a guidance for further evolutions. Experiments on numerical optimization problems indicate that the pheromone based vaccination operator has the ability of acquiring useful information about the objective functions and developing effective vaccines dynamically.

\section{Pheromone Based Vaccination}

The pheromone is a term from ant colonies. Inspired by the foraging behavior of ants, M. Dorigo pioneered the ant colony algorithm (ACA) [9] and applied it to the traveling salesman problem (TSP) [10]. Ant colony algorithm is a novel category of bionic algorithm for optimization problems. It is suitable for solving combinational optimization problems, but has the limitation of stagnation and is easy to fall into local optimums when dealing with massive problems. Moreover, it is difficult for ACA to deal with optimization problems with continuous searching space. However, ACA provides illuminations that can be utilized effectively to learn the developing tendency of genes with continuous coding spaces. Inspired by ACA, a pheromone based vaccination operator is designed for artificial immune algorithms to solve optimization problems with continuous searching space. The optimization problems with continuous searching space can be described as follows:

$$
\min f(\mathbf{x}), \quad \mathbf{x}=\left(x_{1}, x_{2}, \ldots, x_{n}\right) \in S
$$

In formula (1), $f(\mathbf{x})$ is the target function of the optimization problem, $\mathbf{S} \subseteq R^{n}$ is the $n$-dimensional continuous searching space with border $\underline{x_{i}} \leq x_{i} \leq \overline{x_{i}}, i=1,2, \ldots, n$.

\subsection{Real Number Coding and Antibody-Antigen Affinity Definition}

The proposed vaccination operator is designed for real number coded immune algorithms. For an optimization problem with $n$ variables, the antibody coding is a real number string with length $n$, described as, $g_{i} \in[0,1] i=1,2, \ldots, n$. Note $\mathbf{X}=\left(x_{1}, x_{2}, \ldots, x_{n}\right)$ is the variable of the optimization problem dealt with, $\mathbf{A}$ is the antibody coding of the variable $\mathbf{X}$, described by $\mathbf{A}=e(\mathbf{X}) . \mathbf{X}$ is called the decoding of antibody A, described by $\mathbf{X}=e^{-1}(\mathbf{A})$. Then we can get:

$$
x_{i}=\underline{x_{i}}+\left(\overline{x_{i}}-\underline{x_{i}}\right) \times g_{i}, i=1,2, \ldots, n
$$

As antibody's affinity must be a positive value, we construct a negative real function $g(\mathbf{X})$ which is consistent with $f(\mathbf{x})$. In other words, for any two variables $\mathbf{X}_{1}, \mathbf{X}_{2} \in S$, if $g\left(\mathbf{X}_{1}\right)>g\left(\mathbf{X}_{2}\right)$ then $f\left(\mathbf{X}_{1}\right)>f\left(\mathbf{X}_{2}\right)$. In this case, the original 
optimization problem becomes $\min \left\{g\left(e^{-1}(\mathbf{A})\right): \mathbf{A} \in \mathbf{I}\right\}$, in which $\mathbf{I}$ is the antibody space. The antibody-antigen affinity is defined by equation (3):

$$
\operatorname{Affinity}(\mathbf{A})=-g\left(e^{-1}(\mathbf{A})\right)
$$

\subsection{Pheromone Matrix}

From the point of view of each gene location, its value changes from one to another between 0 and 1 to find an appropriate value that maximize affinity of the antibody. Since there are many antibodies in the population, it looks as if there are many ants walk between 0 and 1 to look for food. Therefore, the foraging behavior of ants can be mimicked. The values of genes are continuous, but pheromone concentrations on the pheromone trail can only be recorded discretely. To overcome this issue, we quantize the coding domain of each gene $[0,1]$ into finite number of segments evenly.

For each gene location, an $m$-dimensional real vector is provided to take records of pheromone concentrations, where $m$ is the number of discrete domains. The vector can be described as $\mathbf{P h}_{i}=\left(p h_{1}, p h_{2}, \ldots, p h_{m}\right), \quad p h_{\min } \leq p h_{j} \leq p h_{\max }, 1 \leq j \leq m$, in which $p h_{\min }$ and $p h_{\max }$ are the upper and lower limit of pheromone concentration respectively. As the antibody has $n$ gene locations, these $n$ pheromone vectors make a pheromone matrix with size $n \times m$, noted as $\mathbf{P h}=\left(\mathbf{P h}_{1}, \mathbf{P h}_{2}, \cdots, \mathbf{P h}_{n}\right)^{T}$. It is fulfilled with an initial value of $w$.

\subsection{Vaccination Operators Based on Pheromone}

The pheromone based vaccination proposed in this paper has two parts. One of them is the vaccine injecting operator. In this operator, vaccines will be produced and injected using information from the pheromone matrix. The other one is the pheromone updating operator. It learns useful information from the foregoing evolution by manipulation the pheromone concentration of the pheromone matrix.

\subsubsection{Vaccine Injecting Operator}

The vaccine injection operator will be applied on antibodies of the population after the immune genetic operations as probability $p_{i}$. The vaccine injection operator can be described by the following pseudo code:

$$
\begin{aligned}
& \text { For each antibody of the population } \mathbf{A}_{k}=\left(g^{k}{ }_{1}, g^{k}, \cdots g^{k}{ }_{n}\right) \\
& \text { If }\left\{p_{i}\right\}=\text { TRUE } \\
& \qquad i=\operatorname{Random}(n), \quad j=\operatorname{Location}\left(g^{k}{ }_{i}\right) ; \\
& L L=\left\{\begin{array}{ll}
j-r & \text { if } j-r>0 \\
m+j-r & \text { else }
\end{array}, R=\left\{\begin{array}{ll}
j+r & \text { if } j+r \leq m \\
j+r-m & \text { else }
\end{array}, r=\lfloor m / 8\rfloor ;\right.\right.
\end{aligned}
$$


$t=$ Roulette $(L, R)$;

$$
\begin{aligned}
& \left(g^{k}{ }_{i}\right)^{\prime}=\left\{\begin{array}{ll}
\operatorname{Gauss}\left(g^{k}, \sigma\right) \mid\left[\min _{j}, \max _{j}\right] & \text { if } t=j \\
\text { Latest }(t) & \text { else }
\end{array}, \sigma=10^{-\frac{1}{3} \lg m} ;\right. \\
& g^{k}{ }_{i}=\left(g^{k}{ }_{i}\right)^{\prime} ;
\end{aligned}
$$

End If

\section{End For}

In which, pop_size is the size of the population. The function $\operatorname{Random}(n)$ returns a random integer between 1 and $n$. The function Location $\left(g^{k}{ }_{i}\right)$ returns the serial number of segment in which $g^{k}{ }_{i}$ is located. $\operatorname{Roulette}(L, R)$ is to select a segment between $L$ and $R$ by mean of the roulette selection according to pheromone concentrations. The mutation radius $r=\lfloor m / 8\rfloor$ is the largest integer smaller than $m / 8 \cdot g^{k}{ }_{i}$ and $\left(g^{k}{ }_{i}\right)^{\prime}$ are respectively the gene value before and after the vaccine injection operation. $\operatorname{Gauss}\left(g^{k}, \sigma\right) \mid\left[\min _{j}, \max _{j}\right]$ means gauss mutation with a standard variation of $\sigma$, and it returns a value within the $j$-th segment. In other words, the returned value is between $\min _{j}$ and $\max _{j}$, where $\min _{j}$ and $\max _{j}$ are respectively the lower limit and the upper limit of the $j$-th segment. Latest $(t)$ is the latest gene value that is located in the $t$-th segment. The midpoint value between $\min _{t}$ and $\max _{t}$ will be returned if no gene has previously been assigned to values located in this segment.

\subsubsection{The Pheromone Updating Operator}

For each antibody in the population, apply this operator on it after the vaccine injecting operation. Let $\mathbf{A}$ be any antibody in the population and $\mathbf{A}^{\prime}$ be its offspring after the immune genetic operation and the vaccine injecting operation. The pheromone updating operator can be described by the following pseudo code:

$$
\begin{aligned}
& \text { If } \mathbf{A}^{\prime}=g_{1}^{\prime}, g_{2}^{\prime}, \ldots, g_{n}^{\prime} \text { is superior to } \mathbf{A}=g_{1}, g_{2}, \ldots, g_{n} \\
& \text { For each gene of antibody } \mathrm{A} \\
& \text { If } g_{i} \neq g_{i}^{\prime}, i=1,2, \ldots, n \\
& L s=\operatorname{Location}\left(g_{i}\right), L e=\operatorname{Location}\left(g_{i}^{\prime}\right) ; \\
& \text { Update pheromone of segments between } L s \text { and } L e: \\
& \quad p h^{\prime}=[(1-\rho) p h+\Delta p h] \mid\left[\min _{p h}, \max _{p h}\right], \quad \Delta p h=\frac{Q}{a b s(L e-L s)} ; \\
& \text { Evaporate pheromone of segments beside Ls and Le: } \\
& p h^{\prime}=(1-\rho) p h \mid\left[\min _{p h}, \max _{p h}\right] ;
\end{aligned}
$$




\section{End For}

\section{End If}

In which, $\rho \in(0,1]$ is the pheromone trail decay coefficient. $\mathrm{Q}$ is a real constant and it represents the total amount of pheromone deposed by an ant at ame. The function Location $(g)$ returns the serial number of segment which real number $g$ is located in. The function $\operatorname{abs}(a)$ means the abstract of number $a$. $p h$ and $p h^{\prime}$ are respectively the pheromone concentrations before and after updating. The value of $p h$ and $p h^{\prime}$ must be no smaller than the lower limit $\min _{p h}$ and no larger than the upper limit $\max _{p h}$.

\section{Simulation Experiments}

In order to validate the effectiveness of the proposed vaccination approach, the immune algorithms with pheromone based vaccination are executed to solve the following test functions.

$$
\begin{aligned}
& \text { F01-Min } f(\boldsymbol{x})=\sum_{i=1}^{n} x_{i}^{2} ; \mathcal{S}=[-100,100]^{n} ; f_{\min }=0 ; \\
& \text { F02-Min } f(\boldsymbol{x})=\sum_{i=1}^{n}\left|x_{i}\right|+\prod_{i=1}^{n}\left|x_{i}\right| ; \mathcal{S}=[-10,10]^{n} ; f_{\min }=0 ; \\
& \text { F03-Min } f(\boldsymbol{x})=\max _{i}\left\{\left|x_{i}\right|, 1 \leq i \leq n\right\} ; \mathcal{S}=[-100,100]^{n} ; \quad f_{\min }=0 ; \\
& \text { F04-Min } f(\boldsymbol{x})=\sum_{i=1}^{n}\left(\left\lfloor x_{i}+0.5\right\rfloor\right)^{2} ; \mathcal{S}=[-100,100]^{n} ; \quad f_{\min }=0 ; \\
& \text { F05-Min } f(\boldsymbol{x})=\sum_{i=1}^{n}\left(-x_{i} \sin \left(\sqrt{\left|x_{i}\right|}\right)\right) ; \mathcal{S}=[-500,500]^{n} ; f_{\min }=-418.983 \times n ; \\
& \text { F06-Min } f(\boldsymbol{x})=\sum_{i=1}^{n}\left(x_{i}^{2}-10 \cos \left(2 \pi x_{i}\right)+10\right) ; \mathcal{S}=[-5.12,5.12]^{n} ; f_{\min }=0 ; \\
& \text { F07-Min } f(\boldsymbol{x})=-20 \exp \left(-0.2 \sqrt{\frac{1}{N} \sum^{n} x_{i}^{2}}\right)-\exp \left(\frac{1}{n} \sum_{=1}^{n} \cos \left(2 \pi x_{i}\right)\right)+20+e ; \mathcal{S}=[-30,30]^{n} ; f_{\min }=0 ;
\end{aligned}
$$

F08-Min $f(\boldsymbol{x})=\frac{1}{n} \sum_{i=1}^{n}\left(x_{i}^{4}-16 x_{i}^{2}+5 x_{i}\right) ; \quad \mathcal{S}=[-5,5]^{n} ; \quad f_{\min }=-78.33236 ;$

\subsection{Effectiveness of Pheromone Based Dynamic Vaccination}

Experiments have been done to investigate the effectiveness of pheromone based dynamic vaccination. Table 1 is comparisons of the performance between the clonal selection algorithm (CSA) [2] and the CSA with pheromone based dynamic vaccination (PHDV_CSA). Table 2 is comparisons between the immune programming (IP) [5] and the IP with pheromone based dynamic vaccination (PHDV_IP). All results presented are averages of fifty independent runs. Each run continues until either an optimal solution has been found or the maximum number of generation 1000 is reached. In each run, the control parameters were set as follows: the population size pop_size is 20, the test function dimension $n$ is 10 , the number of segments $m$ is 1000 , the pheromone trail decay coefficient $\rho$ is 0.2 , the constant of total pheromone deposited in one trip $\mathrm{Q}$ is 800 , the initial pheromone concentration $w$ is 1 , the lower and 
upper limit of pheromone concentration are respectively 0.5 and 15 and the vaccine injection probability $p_{i}$ is 0.6 . Other parameters for CSA and IP are the same as those used in reference [2] and [5].

Table 1. Comparison between CSA and PHDV_CSA

\begin{tabular}{c|c|c|c|c}
\hline \multirow{2}{*}{$\begin{array}{c}\text { Test } \\
\text { functions }\end{array}$} & \multicolumn{2}{|c|}{ Average function evaluations } & \multicolumn{2}{c}{ The optimal mean (Standard) } \\
\cline { 2 - 5 } F01 & 26077 & 19074 & $8.13 \times 10^{-4}\left(4.13 \times 10^{-4}\right)$ & $3.21 \times 10^{-4}\left(2.36 \times 10^{-4}\right)$ \\
\hline F02 & 26335 & 22800 & $2.07 \times 10^{-4}\left(1.67 \times 10^{-4}\right)$ & $3.19 \times 10^{-5}\left(4.19 \times 10^{-6}\right)$ \\
\hline F03 & 206936 & 166911 & $7.31 \times 10^{-4}\left(5.66 \times 10^{-4}\right)$ & $3.98 \times 10^{-4}\left(2.14 \times 10^{-4}\right)$ \\
\hline F04 & 17931 & 5414 & $0(0)$ & $0(0)$ \\
\hline F05 & 47839 & 17450 & $-4189.76\left(6.74 \times 10^{-3}\right)$ & $-4189.83\left(3.15 \times 10^{-3}\right)$ \\
\hline F06 & 28619 & 19680 & $5.76 \times 10^{-4}\left(3.36 \times 10^{-4}\right)$ & $1.52 \times 10^{-4}\left(1.17 \times 10^{-4}\right)$ \\
\hline F07 & 30393 & 25306 & $7.31 \times 10^{-4}\left(1.75 \times 10^{-4}\right)$ & $5.18 \times 10^{-5}\left(3.32 \times 10^{-6}\right)$ \\
\hline F08 & 22786 & 7279 & $-78.3304\left(3.29 \times 10^{-5}\right)$ & $-78.3323\left(1.77 \times 10^{-5}\right)$ \\
\hline
\end{tabular}

Table 2. Comparison between IP and PHVV_IP

\begin{tabular}{c|c|c|c|c}
\hline \multirow{2}{*}{$\begin{array}{c}\text { Test } \\
\text { functions }\end{array}$} & \multicolumn{2}{|c|}{ Average function evaluations } & \multicolumn{2}{c}{ The optimal mean (Standard) } \\
\cline { 2 - 5 } & IP & PHDV_IP & IP & PHDV_IP \\
\hline F01 & 29629 & 18524 & $2.84 \times 10^{-4}\left(3.31 \times 10^{-4}\right)$ & $1.95 \times 10^{-4}\left(1.63 \times 10^{-4}\right)$ \\
\hline F02 & 30852 & 20459 & $1.76 \times 10^{-4}\left(3.35 \times 10^{-4}\right)$ & $2.82 \times 10^{-5}\left(3.37 \times 10^{-6}\right)$ \\
\hline F03 & 397596 & 173426 & $7.06 \times 10^{-4}\left(2.17 \times 10^{-4}\right)$ & $5.13 \times 10^{-4}\left(3.39 \times 10^{-4}\right)$ \\
\hline F04 & 22164 & 7096 & $0(0)$ & $0(0)$ \\
\hline F05 & 56580 & 18126 & $-4189.74\left(4.11 \times 10^{-3}\right)$ & $-4189.82\left(2.76 \times 10^{-3}\right)$ \\
\hline F06 & 32460 & 18288 & $3.67 \times 10^{-5}\left(6.17 \times 10^{-5}\right)$ & $1.27 \times 10^{-4}\left(5.42 \times 10^{-4}\right)$ \\
\hline F07 & 36799 & 24421 & $4.92 \times 10^{-4}\left(1.97 \times 10^{-4}\right)$ & $4.55 \times 10^{-5}\left(7.94 \times 10^{-6}\right)$ \\
\hline F08 & 26126 & 9329 & $-78.3306\left(6.71 \times 10^{-4}\right)$ & $-78.3322\left(2.34 \times 10^{-5}\right)$ \\
\hline
\end{tabular}

It can be seen from these data that PHDV_CSA and PHDV_IP perform much better than CSA and IP, as they have found superior solutions with less function evaluations. These data enable us to conclude that the pheromone based dynamic vaccination is effective on improving the searching capability of immune algorithms. Moreover, the comparisons of the standard deviation indicate that the proposed vaccination strategy makes the immune algorithms much more robust.

\subsection{Convergence of Pheromone}

This experiment is designed to investigate whether the pheromone based vaccination approach has learned the real information about the test functions. Considering the first vector of the pheromone matrix, Fig.1 and Fig. 2 show the convergence process of the pheromone distribution while solving the optimization problem of the $30 \mathrm{di}$ mensional test functions F1 and F5 with PHDV_CSA. 

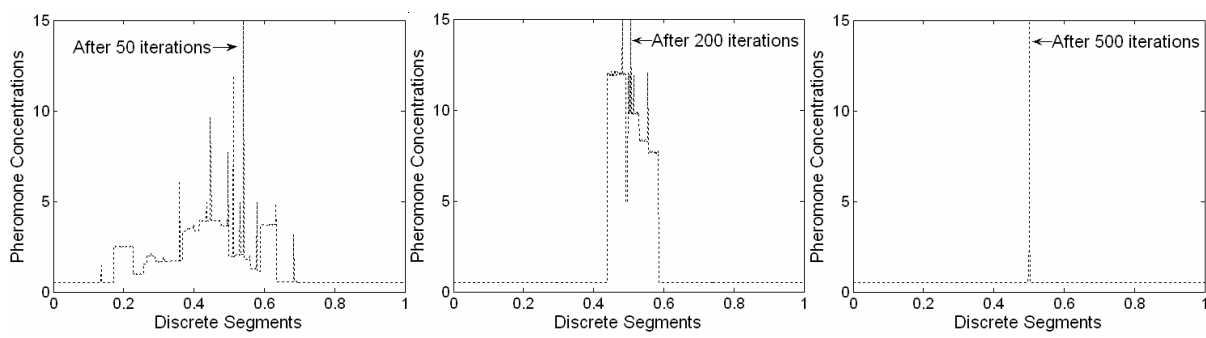

Fig. 1. The change of the pheromone distribution for F1
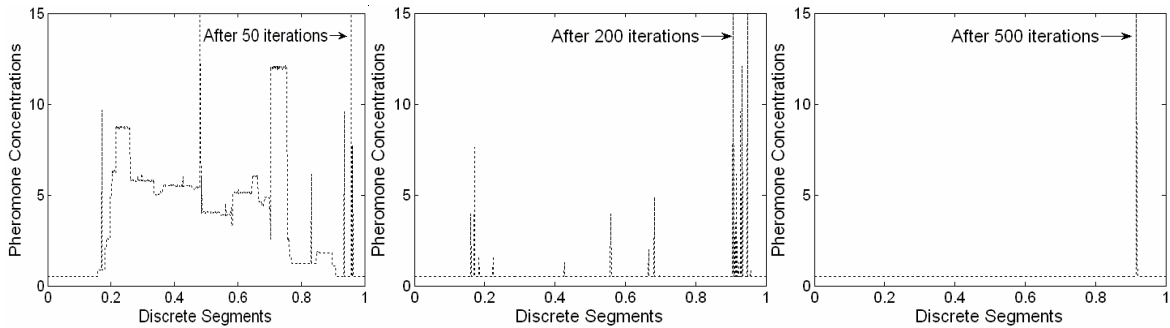

Fig. 2. The change of the pheromone distribution for F5

Take F1 for example, its best value is $x^{*}=(0,0, \cdots, 0)$. The best value in the first dimension is 0 which is mapped to a gene value of 0.5. It can be seen from Fig. 3 that, the pheromone concentrations converge to the segment around the very value of 0.5 . Another example is F5. The best value of the function F5 is $x^{*}=(402.9687,402.9687, \cdots, 402.9687)$. Its best value in first dimension 402.9687 is mapped to a gene value of 0.9029687 in the coding space. Fig.4 indicates that the pheromone concentrations converge to the segment around the very value after 500 iterations. Therefore, we come to the conclusion that the pheromone based vaccination approach has achieved the real information about the objective functions.

\section{Concluding Remarks}

In this paper, we presented a pheromone based vaccination approach for immune algorithms. Our objective was to design a dynamic vaccination strategy which is independent from prior knowledge about the problem for immune algorithms, so that they could be more self-adaptive and easy to use.

Inspired by the ant colony system, the proposed approach learned useful information from population's evolution by stimulating the pheromone deposition and volatilization mechanism of ant colonies. Experimental results indicate that, the distribution of pheromone concentrations reflects the real information about the objective function, and the pheromone based dynamic vaccination is efficient in improving the performance of immune algorithms. 
However, the proposed vaccination is suitable for immune algorithms without crossover operator. How to make a modification so as to cooperate with the crossover operation is one of the future works.

Acknowledgments. This work has been partially supported by the National Natural Science Foundation of China under Grant No. 60372045 and 60133010, and the National Grand Fundamental Research 973 Program of China under Grant No. $2001 C B 309403$.

\section{References}

1. L. N. de Castro, J. Timmis. Artificial Immune Systems: A New Computational Intelligence Approach. Springer-Verlag, Heidelberg, Germany, August 2002.

2. L. N. De Castro, F. J. Von Zuben. The Clonal Selection Algorithm with Engineering Applications, Proceedings of Workshop on Artificial Immune Systems and Their Applications, 2000:36-37.

3. Kim, J. and Bentley, P. J. Towards an Artificial Immune System for Network Intrusion Detection: An Investigation of Dynamic Clonal Selection, Proceedings of Congress on Evolutionary Computation, 2002:1015-1020.

4. Ruochen Liu, Haifeng Du, Licheng Jiao, Immunity Clonal Strategies. Proceedings of the Fifth International Conference on Computational Intelligence and Multimedia Applications. 2003:290.

5. Lei Wang, Jin Pan, Licheng Jiao. The Immune Programming. Chinese Journal of Computers. 2000, 23(8): 806-812.

6. Yanjun Li, Tiejun Wu. A Novel Immune Algorithm for Complex Optimization Problems. Proceedings of the International Conference on Control and Automation. Hangzhou China. June, 2004: 2309-2312.

7. Licheng Jiao, Lei Wang. A Novel Genetic Algorithm Based on Immunity. IEEE Transactions on Systems, Man and Cybernetics. Part A, 2000, 30(9): 1-10.

8. Hua Bo, Fulong Ma, Baojun Han, Licheng Jiao. SAR Image Segmentation based on Immune Algorithm. Proceedings of the International Conference on Control and Automation. Budapest, Hungary. June, 2005: 1279-1282.

9. A. Colorni, M. Dorigo, V. Maniezzo. Distributed optimization by ant colonies. Proceedings of the 1st European Conference on Artificial Life. Pans, France. 1991:134-142.

10. M. Dorigo, L. M. Gambardella. Ant Colony System: A cooperative Learning Approach to the Traveling Salesman Problem. IEEE Transactions on Evolutionary Computation. 1997. 1(1):53-66. 